\title{
BMJ Open Study protocol for a randomised controlled trial with clinical, neurophysiological, laboratory and radiological outcome for surgical versus non-surgical treatment for lumbar spinal stenosis: the Uppsala Spinal Stenosis Trial (UppSten)
}

\author{
Konstantinos Pazarlis, ${ }^{\oplus 1,2}$ Anna Punga, ${ }^{3}$ Nikos Schizas, ${ }^{1,2}$ Bengt Sandén, ${ }^{2}$ \\ Karl Michaëlsson, ${ }^{\circledR 2}$ Peter Försth ${ }^{1,2}$
}

To cite: Pazarlis K, Punga A, Schizas N, et al. Study protocol for a randomised controlled trial with clinical, neurophysiological, laboratory and radiological outcome for surgical versus non-surgical treatment for lumbar spinal stenosis: the Uppsala Spinal Stenosis Trial (UppSten). BMJ Open 2019;9:e030578. doi:10.1136/ bmjopen-2019-030578

- Prepublication history and additional material for this paper are available online. To view please visit the journal (http:// dx.doi.org/10.1136/bmjopen2019-030578).

Received 20 March 2019 Revised 30 July 2019 Accepted 30 July 2019

D Check for updates

(c) Author(s) (or their employer(s)) 2019. Re-use permitted under CC BY-NC. No commercial re-use. See rights and permissions. Published by BMJ.

For numbered affiliations see end of article.

\section{Correspondence to} Dr Konstantinos Pazarlis; pazarlis.konstantinos@gmail. com

\section{ABSTRACT}

Introduction Symptomatic lumbar spinal stenosis is the most common indication for spinal surgery. However, more than one-third of the patients undergoing surgery for lumbar stenosis report dissatisfaction with the results. On the other hand, conservative treatment has shown positive results in some cases. This trial will compare the outcomes of surgical versus non-surgical treatment for lumbar stenosis. The study includes a multidimensional followup, aiming to study the association between outcome and other studied parameters, mainly electromyography and nerve conduction. Moreover, it may contribute to a better understanding of the pathophysiology of lumbar stenosis and to the development of future pharmacologica treatments.

Methods and analysis UppSten is a single-centre randomised controlled trial in which 150 patients with symptomatic lumbar spinal stenosis will be randomised into one of two treatment arms. The patients in the surgical arm will undergo laminectomy; the patients in the non-surgical arm will be given a structured physical training programme. The primary outcome of the study will be the Oswestry Disability Index. Secondary outcomes will include motor amplitude and degree of denervation activity obtained by means of nerve conduction studies and electromyography. Patient-reported outcome measures will be also used as secondary outcomes. Blood sample analysis and the investigation of potential inflammation markers are the additional secondary outcome parameters. Laboratory evaluation will include blood sample collection before the treatment initiation and after 6 months. Flavum ligament biopsies will be performed in the surgical group. Finally, tertiary outcomes will include neurophysiological measures, the objective walking ability and radiological evaluation.

Ethics and dissemination The study is approved by the Local Ethics Committee (Dnr 2017-506), the Hospital's Clinical Trials Committee (2018-0001) and the National Biobank Council and Uppsala Biobank (BbA-827-2018-
Strengths and limitations of this study

- To our knowledge, we plan the first randomised controlled trial comparing surgical and non-surgical treatment for lumbar spinal stenosis that aims to evaluate different clinical dimensions based on clinical, radiological, neurophysiological and laboratory measurement.

- If a neurophysiological examination could predict which patients might benefit from surgery, many patients could potentially avoid surgery and the associated risks.

- Identification of inflammation factors could lead to the development of future pharmacological treatments.

- If non-surgical therapy proves less effective than surgical treatment, there is a risk for crossover.

025). The results will be presented in peer-reviewed journals and at international conferences.

Trial registration number NCT03495661

\section{BACKGROUND}

Lumbar spinal stenosis (LSS) is characterised by low back and leg pain, walking disturbances and in some cases impaired balance and numbness of the lower limbs. This condition is caused by degenerative changes in the lumbar spine, including bulging discs, osteophytes from the arthritic facet joints and thickened flavum ligament (FL), which concertedly cause a narrowing of the spinal canal and compression of lumbar nerve roots. ${ }^{1-3}$ LSS mainly affects populations aged $>65$ years and is unusual in people $<50$ years. ${ }^{4}$ LSS is attracting increasing interest given that 
the world's population is ageing rapidly and increasing demands for physical activity. Today, LSS is the most common indication for spinal surgery among a geriatric population. ${ }^{5-7}$ The goal of surgical treatment in LSS is to decompress the nerve structures in the stenotic segments through a posterior approach. The hypertrophic ligaments and parts of the facet joints are removed (ie, decompression). Adding spinal fusion for stabilisation of the decompressed segment has not been shown to provide superior results compared with decompression alone. ${ }^{8-10}$ Weinstein et al performed a study with 289 patients randomised to either surgical or non-surgical treatment. The authors found that at a 6-month follow-up, the cross-over percentage was up to $36 \%$ and at 1 and 2-year follow-ups, it was more than $40 \% .{ }^{11}$ In contrast, Slätis $\mathrm{et} \mathrm{al}$, who randomised 94 patients to surgical and non-surgical procedures, had a lower cross-over percentage but the difference between the two groups decreased significantly over time and at 6 years there was almost no difference. ${ }^{12}$ Thus, methodological difficulties and the substantial proportion of cross-over effects in the past studies suggest that there is still uncertainty about whether surgery is the most effective treatment approach.

In one study, only $60 \%-70 \%$ of the patients reported to be satisfied with the result after decompression and a few experienced no improvement. ${ }^{13}$ Atlas et al compared surgical and non-surgical treatment for LSS and found similar outcomes between groups at 10 years. ${ }^{14}$ Furthermore, several studies have reported beneficial results for conservative treatment in selected patient groups. ${ }^{15}$ Other studies indicate that any benefits of surgical treatment decrease over time ${ }^{1216}$ and that regular physical exercise may reduce the need for surgery in LSS. ${ }^{17}$ Moreover, surgery, at least in the short term, has substantial positive placebo effects that can improve symptoms in some diseases. ${ }^{18}$

Results from nerve conduction studies (NCSs) and electromyography studies (EMG) have shown that these modalities could have a possible predictive value for the natural course of LSS. ${ }^{19}$ If a neurophysiological examination could predict which patients might benefit from surgery, many patients could potentially avoid surgery and the risks, which would introduce the potential for a personalised medical approach in the treatment of LSS.

Previous trials investigating connective tissue and blood samples have shown that inflammation markers and nociceptors are upregulated in patients with patellar tendinosis, which is an inflammatory condition. ${ }^{20-22}$ Changes in the connective tissue that cause LSS are mainly inflammatory and involve the arthritic facet joints and the FL. ${ }^{23}$ A feasible theory of the pathophysiology of disability and pain related to LSS may involve biological affection of the nerves as a function of inflammatory factors and nociceptors. Identification of some of these factors could lead to a more unifying picture of the pathomechanism underlying nerve compression in LSS and to the development of future pharmacological treatments to be used instead of or in combination with surgery.

\section{Aims}

The null hypothesis is that surgical and non-surgical treatment will be equally effective for patients diagnosed with LSS.

Thus, the primary aim of this study is to evaluate whether surgery with decompression leads to superior results when compared with non-surgical treatment with structured physical therapy. For this evaluation, the Oswestry Disability Index (ODI) ${ }^{24}$ will be used.

The secondary aim is to investigate, by means of NCSs and EMG, whether the degree of neurological affection caused by nerve compression influences the outcome of surgery for LSS.

The questions at issue are these:

1. Does decompression provide a better clinical outcome than non-surgical treatment?

2. Is there a correlation between grade of the clinical symptoms and severity of neurological affection as measured by NCSs/EMG?

3. Are there any connections between neurological deficits and inflammation markers and nociceptors in the blood as well as in immunohistochemical findings from FL? Are these correlated with grade of the clinical symptoms?

4. Does decompression provide superior neurological recovery, as measured by NCSs/EMG, in comparison with non-surgical treatment?

5. Can decompression improve the sagittal balance of the spine?

\section{METHODS}

\section{Trial design}

The Uppsala Spinal Stenosis Trial is a single-centre randomised, controlled clinical trial. All patients participating in the study will be treated at the Uppsala University Hospital. The trial was designed according to the Standard Protocol Items: Recommendations for Interventional Trials reporting guidelines. ${ }^{25} \mathrm{~A}$ flow chart of the trial is displayed in figure 1 .

\section{Sample size calculation}

In all, 150 patients will be included (75 in each treatment arm). We need 40 follow-up patients in each group at a power of $80 \%$, a two-sided alpha of 0.05 and an ability to detect 12 units (SD19) of difference in the ODI. We chose a difference of 12 according to our experience from our previous randomised controlled trials (RCT) within the same setting and since a decrease in the ODI score of 15 had been suggested by the Food and Drug Administration in order to indicate minimally important improvement after spinal fusion surgery. ${ }^{96}$ An SD of 18 units was shown in our prior RCT and we, therefore, chose a slightly higher value (SD 19) in the planning of our new study. ${ }^{9}$ Moreover, experience from previous registry-based RCTs within the Swedish Spine Register (Swespine) regarding lost to follow-up and crossover (ie, patients admitted to non-surgical treatment who wish to switch to 
UppSten Trial

Data collection:

- PROMs

- Walking test

- NCS/EMG

- Standing scoliosis X-ray

- Blood samples

- Pain drawings

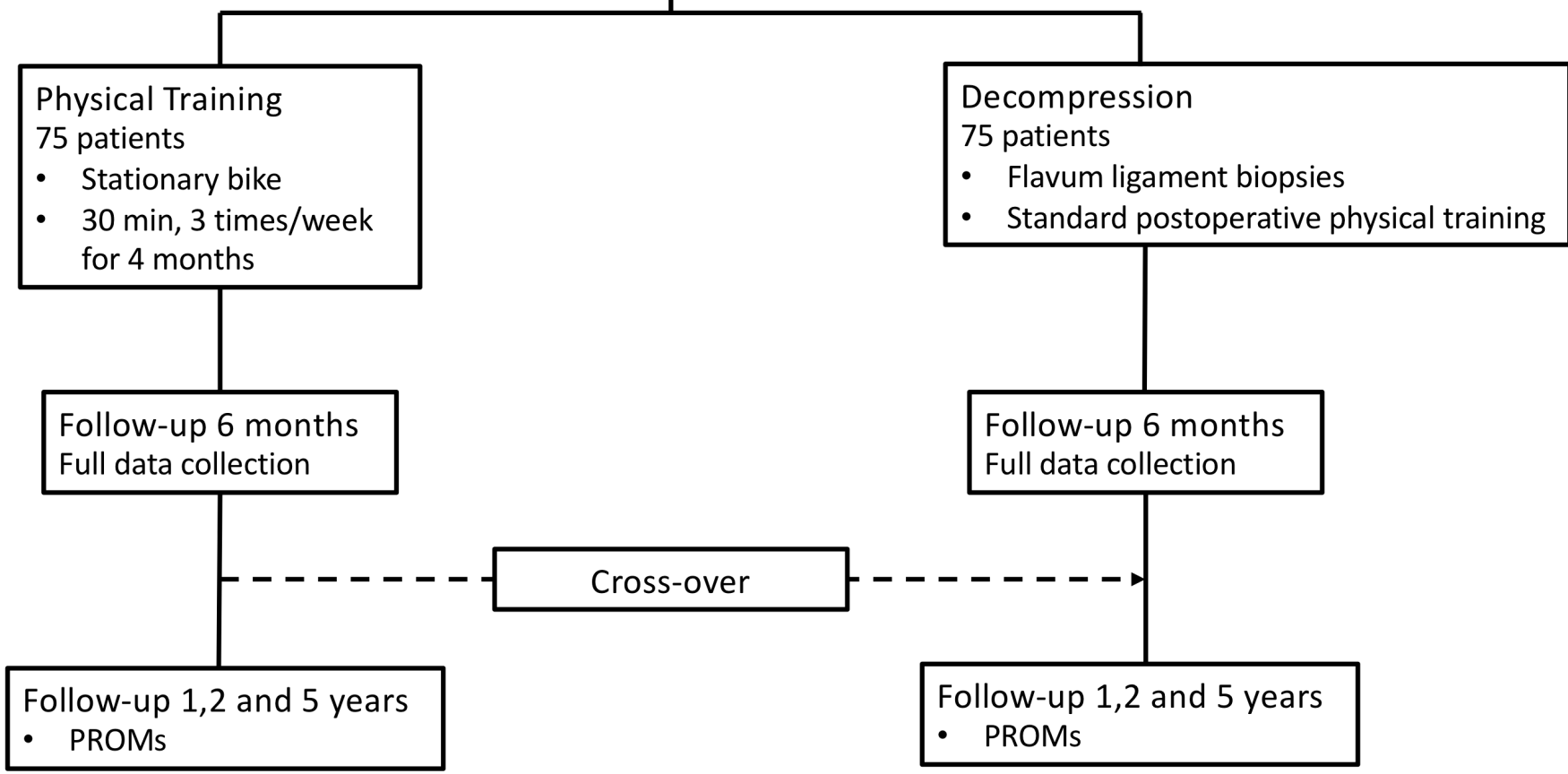

Figure 1 Flow chart of the UppSten trial. EMG, electromyography; NCS, nerve conduction study; PROM, Patient-Reported Outcome Measures.

surgical intervention) is considered. Lost to follow-up is low ( $<5 \%$ after 2 years).$^{8}$ In previous studies, the proportion of patients who crossed over from their treatment assignment varied between $9 \%$ and $40 \%{ }^{11}{ }^{16}$ Lost to follow-up and crossover is estimated to be up to $45 \%$ in our study, which means that 75 patients are needed in each group. An interim analysis will be performed when 100 patients have passed the 6-month follow-up in order to decide if an extended inclusion would be needed due to high crossover. Each year, 100-130 patients, who meet the inclusion criteria, are operated at the Department of Orthopaedics, Uppsala University Hospital. We estimate that 150 patients will be recruited to the study within a period of 24 months.

\section{Statistics}

The t-test will be used to compare the groups for normally distributed variables and logarithmic transformations will be used for non-normally distributed variables. However, in some instances where the transformation may not assure normality, or the outcome is for instance of an ordinal nature, the Mann-Whitney $U$ test will be needed. For variables that are measured both at baseline and at follow-up, analysis of covariance will be used. The primary outcome will be analysed according to the principle of intention-to-treat and to as-treated analysis.
Linear regression models and logistic regression will be used to determine the level of association between clinical grade and neurophysiological parameters. Finally, a mixed-model analysis will be used in order to deal with missing data.

\section{Patients}

A senior orthopaedic spine surgeon will assess the eligibility of all patients aged 50-85 years who are referred to the Department of Orthopaedics at Uppsala University Hospital for LSS. The patients should have a minimum duration of symptoms of 3 months. Inclusion and exclusion criteria are listed in box 1 . The patients who are eligible but not included in the trial will be registered and accounted for as well. If the inclusion criteria are met and after oral and written consent (online supplementary appendix 1), the patients will be randomised into one of the two treatment (surgical or non-surgical) arms.

\section{Inclusion and randomisation}

The participants will be allocated to either arm of the trial using a permuted block randomisation procedure of variable block sizes. The process will be performed by the internet-based randomisation software WebCRF (AKF, Faculty of Medicine and Health Sciences, PO Box 8905 MTFS, NO-7491 Trondheim, Norway). After inclusion, 


\section{Box 1 Inclusion and exclusion criteria}

\section{Inclusion criteria}

1. Age $50-85$ years.

2. Clinical symptoms of lumbar spinal stenosis (pseudoclaudication) indicating and motivating surgery. Numeric Rating Scale in lower $\operatorname{limbs} \geq 3$.

3. MRI with finding of lumbar spinal stenosis at 1-3 lumbar levels. Dural sac area $\leq 75 \mathrm{~mm}^{2}$ or degree of stenosis $C$ or D according to Schizas's classification. ${ }^{34}$

4. The surgical treatment to be provided is decompression alone.

5. The patient has given oral and written informed consent to participate.

\section{Exclusion criteria}

1. Degenerative deformity with Cobb angle $>20^{\circ}$.

2. Spondylolysis.

3. Symptomatic osteoarthritis in the lower limbs that affects and limits the patient's function.

4. Arterial insufficiency (claudication intermittent).

5. Former lumbar surgery other than disc hernia.

6. Conditions that affect the spine, such as ankylosing spondylitis, diffuse idiopathic skeletal hyperostosis, spondylodiscitis/infections, malignancy and neurological diseases.

7. Heart and lung diseases presenting a significant risk for surgery or making it impossible for the patient to take part in a physical training programme (American Society of Anesthesiologists (ASA) score $>3$ ).

8. Polyneuropathies.

9. Psychological factors rendering the patient incapable of inclusion in the trial (eg, drug addiction, dementia).

each participant will receive a code consisting of a letter and a number. The letter indicates the group to which the participant has been assigned and the number corresponds to the allocated ranking within the group. All participant-related documents will be marked with this code. The principal investigator will be responsible for the coding and tagging of the data.

\section{Documentation and data safety}

All healthcare contacts will be documented in accordance with the Department of Orthopaedics, Uppsala University Hospital routine procedures. These documents, together with the identity list and the completed forms, will be stored in folders that will be kept in a locked room with access limited to authorised persons. Research officers ensure that all variables from the completed forms are continuously entered into a database. All data management is done in accordance with the General Data Protection Regulation and Good Clinical Practice.

Once the trial is completed, all material belonging to the study will be stored at the clinic for 1 year and then archived for 25 years according to the department's guidelines for the storage of research material.

\section{Treatment arms}

Group A: Surgery with decompression. Central decompression of the stenotic segment with undercutting of the lateral recesses. The intervention is done through a $3-4 \mathrm{~cm}$ skin incision for one-level, $4-5 \mathrm{~cm}$ for two-level and $6-7 \mathrm{~cm}$ for three-level stenosis. The procedure is done under magnification according to the surgeon's own preference (loupes or microscope). After surgery, free mobilisation is allowed. The follow-up includes instructions for physical training and visits to physiotherapist for regular postoperative training with start 4 weeks after surgery.

Group B: Non-surgical treatment with structured physical training programme, that is, solely static bicycle training according to the 'Östersund model ${ }^{17}$ ( $30 \mathrm{~min}, 3$ times/week for 4 months).

In group A, in conjunction with the surgical procedure, FL will be collected (which is routinely removed during decompression surgery). The ligaments will be examined with immunohistochemical methods for inflammation markers and nociceptors.

\section{Baseline data}

The baseline data that will be collected will include data from demographic and comorbidity variables. Generic and specific outcome measures will be collected from the Swespine. The outcome measures that will be used are the ODI, the EuroQol Five-Dimensional descriptive system questionnaire (EQ-5D), ${ }^{27}$ the Numeric Rating Scale (NRS) ${ }^{28}$ for low back and leg pain, the subjective walking ability tool, the global assessment (GA) instrument $^{2930}$ and the patient satisfaction (PS) questionnaire. The latter two are assessment tools of the Swespine. ${ }^{6} 1330$

Pain drawings, assessment of anxiety using the Hospital Anxiety and Depression Scale ${ }^{31}$ and evaluation of walking ability using the $6 \mathrm{~min}$ walking test (6MWT) will be performed. ${ }^{32}$ In addition, radiological (standing scoliosis plain X-rays), laboratory (blood samples) and neurophysiological measurements (NCSs/EMG) will be assessed in both groups. FL will be evaluated only in the surgical group.

\section{Crossover}

At the 6-month follow-up, there is a possibility of crossover from group B to group A. After crossover to surgery, the after-treatment of these patients will be as in group A.

\section{Radiological evaluation}

The radiological evaluation of the lumbar lordosis (LL) and the sagittal vertical axis (SVA) will be performed by an orthopaedic spine surgeon with more than 5 years of experience in spine surgery. LL will be measured by the Cobb angle between the superior endplate of L1 and the S1 plateau. SVA will be calculated as the distance from the posterior-superior angle of the $\mathrm{S} 1$ to a vertical plumb line dropped from the $\mathrm{C} 7$ centroid.

\section{Laboratory evaluation}

Blood samples will be collected from all patients before treatment and at the 6-month follow-up. In the surgical treatment group (group A), an $1 \times 1 \mathrm{~cm}$ sample of $\mathrm{FL}$ will be dissected and collected from each patient. If the stenosis involves more than one level, the ligament will be collected from the most stenotic segment according to MRI data. If it is not possible to distinguish which level 
is most stenotic, the ligament will be chosen at random. The ligament samples will be examined by immunohistochemistry and proteomic analysis while blood samples will be assessed by proteomic analysis. Immunohistochemistry will be performed at the Orthopaedic laboratory, Uppsala University, Uppsala, Sweden. Proteomic analysis will be performed in collaboration with OLINK proteomics, Uppsala Science Park, Uppsala, Sweden (www.olink. com) according to a predetermined inflammation factor panel consisting of 92 proteins. The panel will include inflammation-related biomarkers such as interleukin-1 alpha (IL, IL-1 beta, IL-1 receptor antagonist, tumour necrosis factor alpha and nociceptors such as glutamate, $\mathrm{N}$-methyl-D-aspartate receptor 1 and substance-P. The exact composition of the panel will be decided on after the enrolment of all patients because at that time point relevant and newly established factors might be of interest (https://www.olink.com/products/inflammation).

The samples will be coded numerically to maintain confidentiality and stored safely and only authorised personnel will have access to the safety room's key code. Sample management will be in accordance with the Ethics Examination Act of Human Research (2003:460) and the rules of the Uppsala Biobank.

\section{Outcomes}

Primary outcome

\section{Oswestry Disability Index}

Secondary outcome

1. Neurophysiology: motor nerve amplitude (NCSs) and degree of denervation activity (EMG).

2. From Swespine: EQ-5D, back pain (NRS), leg pain (NRS), subjective walking ability, GA and PS.

Tertiary outcome

1. Neurophysiology: sensory nerve amplitude, F-response latency and H-reflex (NCSs), number of motor units (MUNIX) and degree of reinnervation (EMG).

2. Objective walking ability (6MWT).

3. Radiological: LL and SVA.

\section{FOLLOW-UP}

Six months, 1, 2 and 5 years

The results at the 2-year follow-up are of main concern for the goal of the study and hence on which the main clinical results will be built. The neurophysiological results will be analysed and presented after the 6-month follow-up. During the follow-up, adverse events (AEs) and serious AEs (SAEs) will be documented for both treatment arms. SAEs are defined as life-threatening events or events that result in death or require hospitalisation or result in significant disability. ${ }^{33}$

\section{Patient and public involvement}

Patients and public were not involved in the design, recruitment and conduction of this study.

\section{Dissemination}

The study, regardless of the outcomes, will be submitted for publication in a peer-reviewed journal. Moreover, the preliminary and final findings will be presented at national and international spinal conferences.

\section{DISCUSSION}

The UppSten study is an RCT with three categories of outcome: primary, secondary and tertiary. To our knowledge, it is the first RCT comparing surgical and non-surgical treatment for LSS that incorporates clinical, neurophysiological, laboratory and radiological evaluation.

The goal of the trial is to study the clinical results of surgical versus non-surgical treatment for LSS. The patients randomised to the surgical arm will undergo a decompressive central laminectomy with undercutting of the lateral recesses.

All patients randomised to the non-surgical arm will undergo a structured physical training programme based on static bicycle training. ${ }^{17}$

One of the secondary aims of the UppSten trial is to examine whether the neurophysiological evaluations (NCSs/EMG) reveal any correlation with the grade of LSS symptoms. Up to now, the standard treatment for patients with symptomatic clinical and radiological spinal stenosis and a duration of symptoms more than 3 months has been surgical decompression. However, it has been speculated whether the compression of the nerve roots causes permanent nerve damage with muscle denervation in some patients, whereas in other patients a reinnervation and recovery of the function may occur.

The degeneration of the lumbar spine progressively impairs spinal sagittal balance. The need to implement extensive corrections and fusion in addition to the decompression to restore sagittal balance continues to be debated among spinal surgeons. The knowledge gained from past RCTs is that patient back pain is reduced by decompression only. ${ }^{8}$ Moreover, according to our experience, many patients report that their posture improved after decompression alone. Thus, the radiological examination intends to help us understand whether decompression surgery can improve the sagittal balance of the spine.

We believe that the UppSten trial will provide useful information about the long-term effects of the two treatment arms for LSS. In addition, the study will examine results from neurophysiological, radiological and immunohistochemical assessments that should help spinal surgeons gain a fuller understanding of the pathophysiology of LSS and formulate more accurate predictions as to which patients have better chances of benefiting from surgery. Up to now (March 2019), 51 patients have been included in the trial.

\section{Author affiliations}

${ }^{1}$ Spine Surgery Unit, Department of Orthopaedics, Uppsala University Hospital, Uppsala, Sweden

${ }^{2}$ Surgical Sciences, Division of Orthopaedics, Uppsala University, Uppsala, Sweden ${ }^{3}$ Neuroscience, Division of Clinical Neurophysiology, Uppsala University, Uppsala, Sweden 
Contributors KP and PF wrote the protocol KP, PF and AP designed the study. KP, $\mathrm{PF}, \mathrm{AP}, \mathrm{NS}, \mathrm{BS}$ and KM critically reviewed the manuscript.

Funding This study is funded by an Uppsala institutional Avtal om Läkarutbildning och Forskning - ALF (Agreement concerning Cooperation on Medical Education and Research).

Competing interests KP reports grants from Johnson \& Johnson, during the conduct of the study.

Patient consent for publication Written patient consent will be obtained from all patients in the trial.

Ethics approval This trial has been approved by the Local Ethics Committee (Dnr 2017-506), the Hospital's Clinical Trials Committee (2018-0001) and the National Biobank Council and Uppsala Biobank (BbA-827-2018-025). UppSten will be conducted in full compliance with the Declaration of Helsinki.

Provenance and peer review Not commissioned; externally peer reviewed.

Open access This is an open access article distributed in accordance with the Creative Commons Attribution Non Commercial (CC BY-NC 4.0) license, which permits others to distribute, remix, adapt, build upon this work non-commercially, and license their derivative works on different terms, provided the original work is properly cited, appropriate credit is given, any changes made indicated, and the use is non-commercial. See: http://creativecommons.org/licenses/by-nc/4.0/.

\section{REFERENCES}

1. Ciricillo SF, Weinstein PR, stenosis Lspinal. Lumbar spinal stenosis. West J Med 1993;158:171-7.

2. Yong-Hing K, Kirkaldy-Willis WH. The pathophysiology of degenerative disease of the lumbar spine. Orthop Clin North Am 1983;14:491-504.

3. Kirkaldy-Willis WH, Wedge $\mathrm{JH}$, Yong-Hing $\mathrm{K}$, et al. Pathology and pathogenesis of lumbar spondylosis and stenosis. Spine 1978;3:319-28

4. Kalichman L, Cole R, Kim DH, et al. Spinal stenosis prevalence and association with symptoms: the Framingham study. Spine $J$ 2009;9:545-50.

5. Bae HW, Rajaee SS, Kanim LE. Nationwide trends in the surgical management of lumbar spinal stenosis. Spine 2013;38:916-26.

6. Strömqvist B, Fritzell $P$, Hägg $O$, et al. The Swedish spine register: development, design and utility. Eur Spine J 2009;18:294-304.

7. Deyo RA, Gray DT, Kreuter W, et al. United States trends in lumbar fusion surgery for degenerative conditions. Spine 2005;30:1441-5.

8. Forsth P, Michaelsson K, Sanden B, et al. Does fusion improve the outcome after decompressive surgery for lumbar spinal stenosis? A two-year follow-up study involving 5390 patients. Bone $\mathrm{Jt} J$ 2013;95:960-5.

9. Försth P, Ólafsson G, Carlsson T, et al. A randomized, controlled trial of fusion surgery for lumbar spinal stenosis. N Engl J Med 2016;374:1413-23.

10. Sigmundsson FG, Jönsson B, Strömqvist B. Outcome of decompression with and without fusion in spinal stenosis with degenerative spondylolisthesis in relation to preoperative pain pattern: a register study of 1,624 patients. Spine J 2015;15:638-46.

11. Weinstein JN, Tosteson TD, Lurie JD, et al. Surgical versus nonsurgical therapy for lumbar spinal stenosis. N Engl J Med 2008;358:794-810.
12. Slätis $P$, Malmivaara $A$, Heliövaara $M$, et al. Long-Term results of surgery for lumbar spinal stenosis: a randomised controlled trial. Eur Spine J 2011;20:1174-81.

13. Strömqvist $B$, Fritzell $P$, Hägg $O$, et al. Swedish Society of spinal surgeons. Swespine: the Swedish spine register. Eur Spine J 2013;22:953-74.

14. Atlas SJ, Keller RB, Wu YA, et al. Long-Term outcomes of surgical and nonsurgical management of lumbar spinal stenosis: 8 to 10 year results from the Maine lumbar spine study. Spine 2005;30:936-43.

15. Delitto A, Piva SR, Moore CG, et al. Surgery versus nonsurgical treatment of lumbar spinal stenosis. Ann Intern Med 2015;162:465-73.

16. Malmivaara A, Slätis $P$, Heliövaara $M$, et al. Surgical or nonoperative treatment for lumbar spinal stenosis? A randomized controlled trial. Spine 2007;32:1-8.

17. Nord T. Träning minskade behovet AV operation Vid lumbal spinal stenos Belastning AV cirkulationsapparaten I form AV cykling gav Bra effekt. 2015:7-10.

18. Harris I. Surgery, the ultimate placebo. 1st edn, 2016: 80-112.

19. Micankova Adamova B, Vohanka S, Dusek L, et al. Prediction of long-term clinical outcome in patients with lumbar spinal stenosis. Eur Spine J 2012;21:2611-9.

20. $\varnothing \mathrm{L}$, Dahl J, Ackermann PW, et al. Pronociceptive and antinociceptive neuromediators in patellar tendinopathy. Am J Sports Med 2006;34:1801-8.

21. Schizas N, Lian Ø, Frihagen F, et al. Coexistence of up-regulated NMDA receptor 1 and glutamate on nerves, vessels and transformed tenocytes in tendinopathy. Scand J Med Sci Sports 2010;20:208-15.

22. Schizas N, Weiss R, Lian Øystein, et al. Glutamate receptors in tendinopathic patients. J Orthop Res 2012;30:1447-52.

23. Genevay S, Atlas SJ, stenosis Lspinal. Lumbar spinal stenosis. Best Pract Res Clin Rheumatol 2010;24:253-65.

24. Fairbank JC, Pynsent PB. The Oswestry disability index. Spine 2000;25:2940-53.

25. Chan AW, Tetzlaff JM, Altman DG, et al. Spirit 2013 statement: defining standard protocol items for clinical trials. Vol. 158, Annals of internal medicine. American College of Physicians 2013:200-7.

26. Roland M, Fairbank J. The Roland-Morris disability questionnaire and the Oswestry disability questionnaire. Spine 2000;25:3115-24.

27. Herdman M, Gudex C, Lloyd A, et al. Development and preliminary testing of the new five-level version of EQ-5D (EQ-5D-5L). Qual Life Res 2011;20:1727-36.

29. Haefeli M, Elfering A. Pain assessment. Eur Spine J 2006;15(Suppl 1):S17-24.

29. Hägg O, Fritzell P, Odén A, et al. Simplifying outcome measurement: evaluation of instruments for measuring outcome after fusion surgery for chronic low back pain. Spine 2002;27:1213-22.

30. Parai C, Hägg O, Lind B, et al. The value of patient global assessment in lumbar spine surgery: an evaluation based on more than 90,000 patients. Eur Spine J 2018;27:554-63.

31. Zigmond AS, Snaith RP. The hospital anxiety and depression scale. Acta Psychiatr Scand 1983;67:361-70.

32. ATS Committee on Proficiency Standards for Clinical Pulmonary Function Laboratories. Ats statement: guidelines for the six-minute walk test. Am J Respir Crit Care Med 2002;166:111-7.

33. Gliklich RE, Dreyer NA, Leavy MB. Registries for Evaluating Patient Outcomes. Registries for Evaluating Patient Outcomes: A User's Guide. Agency for Healthcare Research and Quality, 2014.

34. Schizas C, Theumann N, Burn A, et al. Qualitative grading of severity of lumbar spinal stenosis based on the morphology of the dural sac on magnetic resonance images. Spine 2010;35:1919-24. 